\title{
含羰基有机添加剂对 $\mathrm{AlCl}_{3}$-[Emim] $\mathrm{Cl}$ 电沉积铝的影响
}

\author{
冷明浩 $a, b$ 陈仕谋 ${ }^{b}$ 张军玲 ${ }^{b}$ 郎海燕 ${ }^{b}$ 康艳红 ${ }^{b}$ 张锁江 ${ }^{*, b}$ \\ ( ${ }^{a}$ 沈阳师范大学 化学与生命科学学院 沈阳 110034) \\ $\left({ }^{b}\right.$ 中国科学院过程工程研究所 绿色过程与工程院重点实验室 北京 100190)
}

\begin{abstract}
摘要 以 $[\mathrm{Emim}] \mathrm{Cl} / \mathrm{AlCl}_{3}(33.3 / 66.7 \mathrm{~mol} \%)$ 离子液体为电解质, 选取了丙酮、乙酰胺、乙酸、乙酸甲酯、氨基甲酸甲酯 5 种含有羰基官能团的有机分子作为添加剂, 讨论其对铝沉积层的影响. 通过 CV 曲线、SEM、XRD、UV-Vis、NMR 等 分析, 进一步研究了添加剂对 $\mathrm{Al}$ 沉积层形貌、晶面取向及沉积机理的影响. 结果表明: 氨基甲酸甲酯是一种性能优异 的整平添加剂, $45 \mathrm{mmol} / \mathrm{L}$ 氨基甲酸甲酯的加入明显改善 $\mathrm{Al}$ 产品的光亮度, 得到细致均匀且镜面光亮的 $\mathrm{Al}$ 沉积层. 氨 基甲酸甲酯为添加剂时, 在电解液体系中没有形成新的金属络合离子, 不影响电解液中活性铝离子结构; 其炭基碳原 子为正电中心在阴极表面吸附, 对 $\mathrm{Al}$ 的电沉积过程产生抑制进而获得整平和光亮效果.
\end{abstract}

关键词＼cjkstart离子液体; 电沉积; 羊基; 氨基甲酸甲酯; 添加剂

\section{Effects of Organic Additives Containing Carbonyl Group on Electrodeposition of $\mathrm{Al}$ from $\mathrm{AlCl}_{3}-[\mathrm{Emim}] \mathrm{Cl}$ Ionic Liquid}

\author{
Leng, Minghao ${ }^{a, b} \quad$ Chen, Shimou $^{b} \quad$ Zhang, Junling $^{b} \quad$ Lang, Haiyan $^{b} \quad$ Kang, Yanhong ${ }^{b}$ \\ Zhang, Suojiang*,b \\ $\left({ }^{a}\right.$ College of Chemistry and Life Science, Shenyang Normal University, Shenyang 110034) \\ ( ${ }^{b}$ Institute of Process Engineering, Chinese Academy of Sciences, Beijing 100190)
}

\begin{abstract}
Electrodeposition of $\mathrm{Al}$ in ionic liquids for preparation of aluminum products had opened up a new research direction, but the previous studies found that aluminum product from pure ionic liquid electrodeposition was unable to meet the requirements in both uniformity and smoothness. Electrodepostion in pure ionic liquid often results in the deposits with rough surface or even dendritic products after long-time deposition. In this study, five kinds of small organic molecules containing carbonyl as additives were investigated, such as acetone, acetic acid amide, acetic acid, methyl acetate and methyl carbamate. Additives were added to $[\mathrm{Emim}] \mathrm{Cl} / \mathrm{AlCl}_{3}(33.3 / 66.7 \mathrm{~mol} \%)$ with a concentration of $5 \mathrm{mmol} / \mathrm{L}, 15 \mathrm{mmol} / \mathrm{L}, 45 \mathrm{mmol} / \mathrm{L}$. Cyclic voltammetry was studied on a glassy carbon electrode at a scan rate of $100 \mathrm{mV} \cdot \mathrm{s}^{-1}$ and temperature of $323 \mathrm{~K}$. The electrode potential was scanned from the open circuit potential to negative-potential, then retraced to get cyclic voltammetry curve. Among those organic additives, methyl carbamate was proved to be an excellent additive. The brightness of $\mathrm{Al}$ can be improved by adding $45 \mathrm{mmol} / \mathrm{L}$ methyl carbamate. Smooth, uniform and mirror bright $\mathrm{Al}$ deposition would be obtained afterward. Furthermore, the effects of additive for $\mathrm{Al}$ deposit on morphology, crystal orientation and $\mathrm{Al}$ deposition mechanism by the analysis of galvanostatic deposition, scanning electron microscope (SEM), X-ray diffraction (XRD), ultraviolet-visible spectroscopy (UV-Vis) and nuclear magnetic resonance (NMR) was conjectured. The results showed that methyl carbamate as an additive would make grain refinement more apparent and present more strong $\mathrm{Al}(200)$ crystal plane orientation. As an additive, methyl carbamate did not form new metal complex in the electrolytic liquid and did not affect the structure of active aluminum ion in the electrolyte. The positive center of carbonyl carbon atom in methyl carbamate molecular can be easily adsorbed at the cathode surface. This procedure inhibited the process of $\mathrm{Al}$ deposition and obtained the smooth and bright effect. Therefore, $\mathrm{Al}$ deposition layer of mirror can be obtained, which has an important guiding significance in the selection of ionic liquid additive in electrodeposition system.

Keywords ionic liquids; electrodeposition; carbonyl; methyl carbamate; additives
\end{abstract}

\section{1 引言}

离子液体具有许多独特的功能, 如低蒸气压, 良好
的离子导电性和较宽的电化学窗口, 并且可设计性强 等 ${ }^{[1 \sim 4]}$, 使得离子液体中电沉积铝等活泼金属成为近年

\footnotetext{
*E-mail: sjzhang@home.ipe.ac.cn

Received December 12, 2014; published April 1, 2015.
}

Supporting information for this article is available free of charge via the Internet at http://sioc-journal.cn.

Project supported by the National Key Basic Research Program of China (No. 2015CB251401), the Key Technologies R\&D Program (No. 2012BAF03B01), the Special Funds of the National Natural Science Foundation of China (No. 21127011) and the General Program of National Natural Science Foundation of China (No. 51274181).

项目受国家重点基础研究发展计划(No. 2015CB251401), 国家科技支撑计划(No. 2012BAF03B01)和国家自然科学基金项目(No. 21127011, 51274181) 资助. 
来的研究热点. Hurley 和 Wier ${ }^{[5]}$ 首次从乙基吡啶溴盐与 三氯化铝的混合物获得的氯铝酸离子液体中电沉积出 铝单质. 此后, 咪唑类 ${ }^{[6 \sim 12]}$ 、季铵盐类 ${ }^{[13,14]}$ 、吡咯类 ${ }^{[15 \sim 19]}$ 氯铝酸离子液体中电沉积铝的研究相继被报道. 其中, 1-乙基-3-甲基咪唑氯化物与三氯化铝混合后得到的氯 铝酸离子液体 $\left([\mathrm{Emim}] \mathrm{Cl} / \mathrm{AlCl}_{3}\right)$ 在室温条件下呈液体状 态, 且具有较宽的电化学窗口, 较高的电导率和较低的 粘度, 被认为是最有望实现工业应用的铝沉积电解液. 在氯铝酸离子液体中, 其活性离子 $\mathrm{Al}_{2} \mathrm{Cl}_{7}^{-}$被电化学还 原，从而获得单质铝.

咪唑类氯铝酸离子液体中电沉积铝研究发现, 纯离 子液体中获得的铝沉积层无法兼顾均匀性和平整性, 表 面不够光滑并且沉积层容易形成枝晶, 因此对产品形貌 具有较好调节作用的电解液添加剂研究成为一个重要 方向 ${ }^{[20,21]}$. 但是相对于水溶液体系, 离子液体中添加剂 的研究还处于起步阶段. Abbott 等 ${ }^{[22]}$ 发现在离子液体中 添加剂 $\mathrm{LiCl}$ 有利于大颗粒 $\mathrm{Al}$ 沉积层的形成, 难以获得 整平效果. $\mathrm{Liu}$ 等 ${ }^{[23]}$ 发现 $\mathrm{LaCl}_{3}$ 有助于得到均匀致密的 $\mathrm{Al}$ 沉积层. $\mathrm{Li}$ 等 ${ }^{[24]}$ 研究了在 $[\mathrm{Emim}] \mathrm{Cl} / \mathrm{AlCl}_{3}$ 中利用脉冲 的方法对铝沉积层形貌的影响, 加入 $\mathrm{NdCl}_{3}$ 后发现沉积 层形貌从多边形变为球状晶体. Barchi 等 ${ }^{[25]}$ 则以 1,10 -邻 二氮杂菲为添加剂在离子液体中电沉积得到了耐腐蚀 性良好的光亮 $\mathrm{Al}$ 沉积层. Endres 等 ${ }^{[26]}$ 以烟酸为添加剂从 $[\mathrm{Emim}] \mathrm{Cl} / \mathrm{AlCl}_{3}(45 / 55 \mathrm{~mol} \%)$ 离子液体中制备出了纳米 级铝晶体. Zhang 等 ${ }^{[27]}$ 以烟酸甲酯为添加剂同样得到了 光亮的 $\mathrm{Al}$ 沉积镀层.

显然, 在离子液体中可通过加入适当的添加剂改进 和影响 $\mathrm{Al}$ 沉积层的形貌和质量. 但现有的离子液体添 加剂方面的研究还集中在无机卤盐类和有机大分子类. 事实上, 有机物小分子在水溶液电解质体系中也是常见 的添加剂, 因为有机小分子具有相对分子质量较小, 添 加到离子液体中不会影响电解质粘度, 电导率也不会降 低等特点. 考虑到羰基为吸电子官能团, 在电极表面具 有合适的吸附能力, 本文以含有羰基基团的有机小分子 作为研究对象, 分析其在 $[\mathrm{Emim}] \mathrm{Cl} / \mathrm{AlCl}_{3}(33.3 / 66.7$ $\mathrm{mol} \%$ )中对 $\mathrm{Al}$ 沉积层表面形貌, 晶体取向和沉积影响, 选取了较优的添加剂并分析了其作用机理.

\section{2 结果与讨论}

\section{1 添加剂的篮选}

研究选取了丙酮、乙酰胺、乙酸、乙酸甲酯、氨基 甲酸甲酯 5 种含羰基的有机小分子作为电解液添加剂, 将其加入 $[\mathrm{Emim}] \mathrm{Cl} / \mathrm{AlCl}_{3}(33.3 / 66.7 \mathrm{~mol} \%$ )中, 浓度分别 为 $5 \mathrm{mmol} / \mathrm{L} 、 15 \mathrm{mmol} / \mathrm{L} 、 45 \mathrm{mmol} / \mathrm{L}$. 首先进行了玻碳 电极上的循环伏安 $(\mathrm{CV})$ 研究, 扫描速率为 $100 \mathrm{mV} \cdot \mathrm{s}^{-1}$, 温度为 $323 \mathrm{~K}$, 无搅拌, 从开路电位向负电位扫描, 再 回扫得到循环伏安曲线, 丙酮、乙酰胺、乙酸、乙酸甲
酯、氨基甲酸甲酯作为添加剂体系的 CV 曲线分别见图 $1 \mathrm{a} \sim 1 \mathrm{e}$. 图 1(a)是添加不同浓度丙酮后获得的 CV 曲线, 铝的还原峰值在 $-0.6 \mathrm{~V}$ 左右, 当加入 $5 \mathrm{mmol} / \mathrm{L}$ 丙酮后, $\mathrm{CV}$ 曲线没有明显变化; 浓度逐渐升高, 达到 $15 \mathrm{mmol} / \mathrm{L}$ 后, $\mathrm{Al}$ 还原峰发生变化, 在一 $-0.6 \mathrm{~V}$ 处发生波动, 初步怀 疑丙酮在离子液体中发生副反应所致; 当浓度值达到 $45 \mathrm{mmol} / \mathrm{L}$ 时, 出现一个峰值较强的还原峰, 证明是丙 酮在离子液体中发生新的反应，不适合作为添加剂使 用. 图 1(b)中加入 $5 \mathrm{mmol} / \mathrm{L}$ 乙酰胺后, 铝还原峰位置向 负方向移动，过电位少量增加; 加入 $15 \mathrm{mmol} / \mathrm{L}$ 时，过 电位没有增加，反而向正向移动; 加入 $45 \mathrm{mmol} / \mathrm{L}$ 后， 也有新的还原峰出现, 有副反应发生, 所以乙酰胺也不 是较好的添加剂选择. 图 1(c)所示, 加入 $5 \mathrm{mmol} / \mathrm{L} 、 15$ $\mathrm{mmol} / \mathrm{L}$ 的乙酸后, 还原峰位置没有变化, 峰值电流有 所降低; 加入 $45 \mathrm{mmol} / \mathrm{L}$ 的乙酸后, 峰位置稍微向负方 向移动, 峰值电流继续降低. 图 1(d)所示, 加入乙酸甲 酯后, $\mathrm{CV}$ 曲线没有明显变化. 图 1(e)所示, 加入氨基甲 酸甲酯后，还原峰位置明显向负方向移动，过电位明显 增加, 过电位的增加通常有利于沉积晶粒的细化和沉积 层的平整光滑作用. 因此，后续选择氨基甲酸甲酯为添 加剂进行系统研究.

\section{2 氨基甲酸甲酯作为添加剂对沉积层影响}

在 $323 \mathrm{~K}$, 电流密度 $1.4 \mathrm{~A} / \mathrm{dm}^{2}$, 无搅拌条件下, 加 入不同浓度 $0 \mathrm{mmol} / \mathrm{L} 、 5 \mathrm{mmol} / \mathrm{L} 、 45 \mathrm{mmol} / \mathrm{L}$ 氨基甲酸 甲酯后, 电沉积 $3 \mathrm{~min}$ 得到 $\mathrm{Al}$ 层照片和 SEM 照片如图 2 和图 3 所示. 宏观由图 2(a)可知, [Emim $] \mathrm{Cl} / \mathrm{AlCl}_{3}$ 无添 加剂时不会得到光亮的铝镀层, $\mathrm{Al}$ 沉积层的光亮度和平 整度都有待提高. 微观由图 3(a)可知, 无添加剂条件下, 晶粒尺寸较大, 晶粒高低不一, 同时存在缝隙, 平整度 较差, 所以得不到光亮 $\mathrm{Al}$ 沉积层. 由图 2(b)可以看出, 添加 $5 \mathrm{mmol} / \mathrm{L}$ 氨基甲酸甲酯后, 铝沉积层光亮度得到 了提高, 可以反射出图中的文字. 由 $\mathrm{CV}$ 曲线可知, 氨 基甲酸甲酯的添加增加了 $\mathrm{Al}$ 沉积的过电位，沉积电位 向负方向移动，有着减小晶粒尺寸的作用. 由图 3(b)可 以看出, $\mathrm{Al}$ 沉积层晶粒分布细致均匀, 同时没有空隙出 现, 整平效果也较为明显, 从而得到了光亮 $\mathrm{Al}$ 沉积层所 以反射文字较为清晰. 由图 2(c)可以看出当氨基甲酸甲 酯浓度达到 $45 \mathrm{mmol} / \mathrm{L}$ 时, 反射出图中的文字更为清晰, 得到的沉积层出现镜面效果. 图 3(c) 可知, 添加剂浓度 的提高使得晶粒细化更为明显, 为镜面效果提供了首要 条件. 分布更加均匀, 晶粒之间紧密排列, 整平效果更 加明显，因此在高电流密度的影响下得到了光亮的镜面 Al 沉积层.

添加剂的加入使得 $\mathrm{Al}$ 沉积电压变得更负, 过电位 增加; 继续加入, 沉积电压变负程度减小, 但是整平光 亮效果提高, 晶粒细化程度加强, 整平光亮效果也较为 提高. 

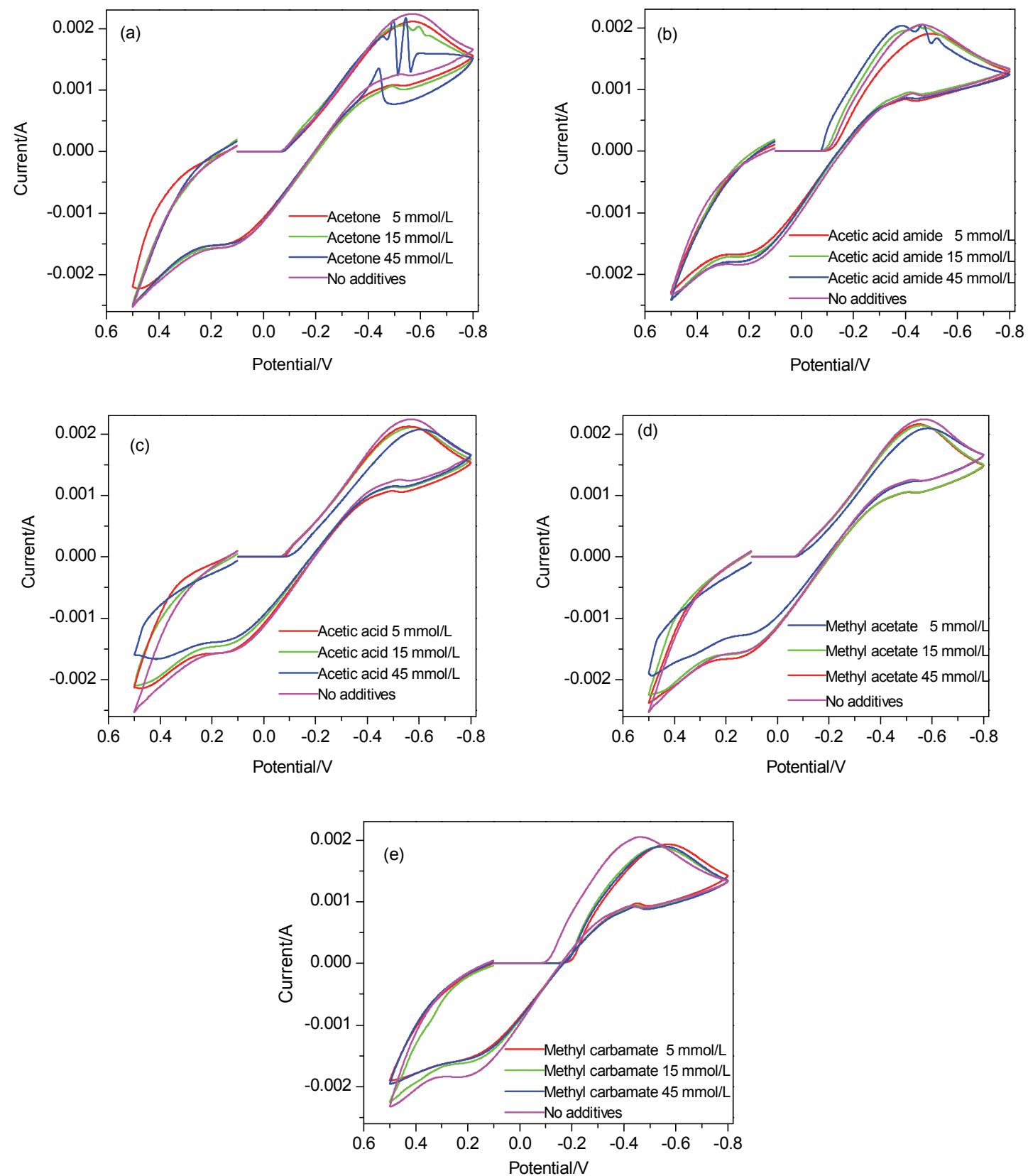

图 1 [ Emim] Cl/ $/ \mathrm{AlCl}_{3}(33.3 / 66.7 \mathrm{~mol} \%$ )中分别加入浓度为 $5 \mathrm{mmol} / \mathrm{L} 、 15 \mathrm{mmol} / \mathrm{L} 、 45 \mathrm{mmol} / \mathrm{L}$ 的丙酮(a), 乙酰胺(b), 乙酸(c), 乙酸甲酯(d)和氨基甲 酸甲酯(e)添加剂在玻碳电极上的循环伏安曲线

Figure 1 Cyclic voltammograms recorded on a glassy carbon electrode in $[\mathrm{Emim}] \mathrm{Cl} / \mathrm{AlCl}_{3}(33.3 / 66.7 \mathrm{~mol} \%)$ containing different concentrations of (a) acetone, (b) acetic acid amide, (c) acetic acid, (d) methyl acetate and (e) methyl carbamate

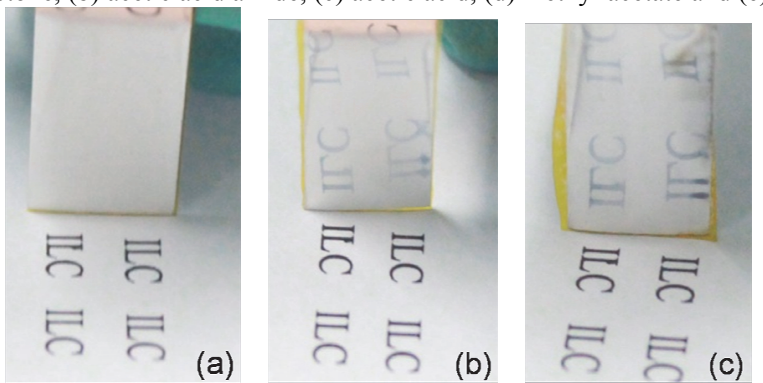

图 2 在 $1.4 \mathrm{~A} / \mathrm{dm}^{2}$ 的电流密度下, 加入不同浓度 $0 \mathrm{mmol} / \mathrm{L} 、 5 \mathrm{mmol} / \mathrm{L}$ 、 $45 \mathrm{mmol} / \mathrm{L}$ 氨基甲酸甲酯后, 电沉积 $3 \mathrm{~min}$ 后获得 $\mathrm{Al}$ 沉积层照片

Figure 2 Photo of the Al deposits from $[\mathrm{Emim}] \mathrm{Cl} / \mathrm{AlCl}_{3}(33.3 / 66.7$ mol\%) containing (a) no additives, (b) $5 \mathrm{mmol} / \mathrm{L}$ methyl carbamate and (c) $45 \mathrm{mmol} / \mathrm{L}$ methyl carbamate at $1.4 \mathrm{~A} / \mathrm{dm}^{2}$
在 $323 \mathrm{~K}$, 无搅拌条件下, 不同浓度 $0 \mathrm{mmol} / \mathrm{L} 、 5$ $\mathrm{mmol} / \mathrm{L} 、 45 \mathrm{mmol} / \mathrm{L}$ 氨基甲酸甲酯添加后的电解液中, 电流密度为 $0.7 \mathrm{~A} / \mathrm{dm}^{2}$, 电沉积 $8000 \mathrm{~s}$ 得到 $\mathrm{Al}$ 层 SEM 照 片如图 4 所示. 由图 4(a)可以看出[ $\mathrm{Emim}] \mathrm{Cl} / \mathrm{AlCl}_{3}$ 无添 加剂电沉积 $8000 \mathrm{~s}$ 所得的 $\mathrm{Al}$ 晶粒尺寸较大, 为不规则 多面体; 由于叠层生长, 晶粒之间有空隙生成, 平整度 一般, 并且容易形成枝晶. 由图 4(b) 可以看出, 加入少 量氨基甲酸甲酯添加剂后, 晶粒尺寸明显减小, 但没有 均匀紧密排列, 还是存在空隙. 如图 4(c)所示, 提高添 加剂浓度达到 $45 \mathrm{mmol} / \mathrm{L}$ 时, 晶粒尺寸相对于低浓度添 加剂没有明显变化, 但是晶形由无规则晶型变为较为规 
则的多面体结构; 晶粒均匀紧密排列, 整平性好, 不易 形成枝晶. 所以即使长时间电沉积, 通过氨基甲酸甲酯 的添加, 也可以得到平滑致密的 $\mathrm{Al}$ 沉积层.
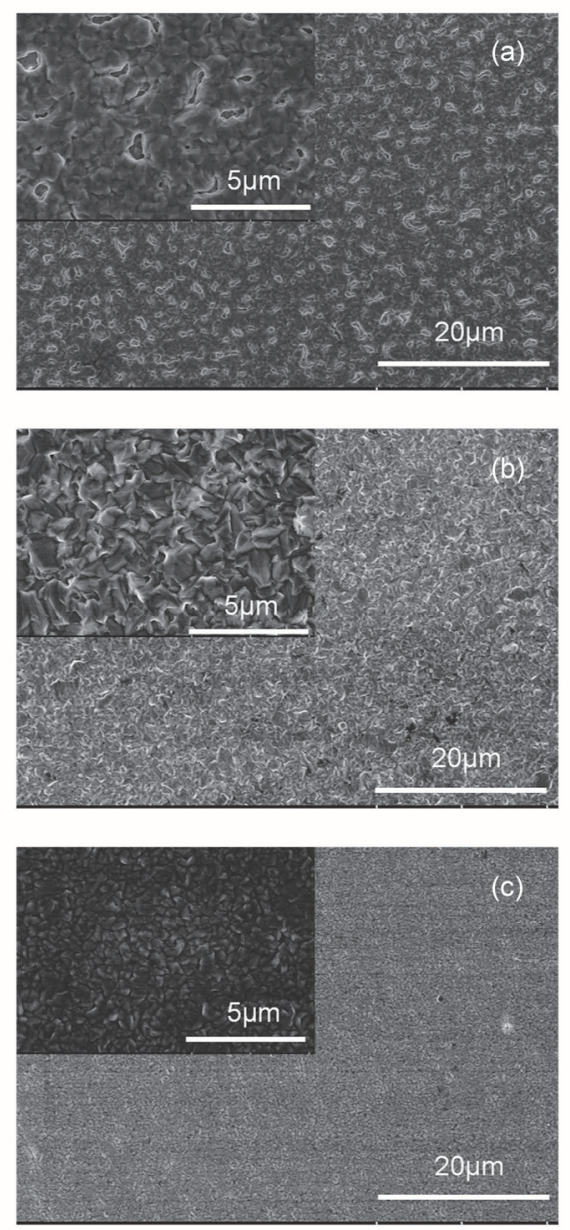

图 3 在 $1.4 \mathrm{~A} / \mathrm{dm}^{2}$ 的电流密度下, 加入不同浓度 $0 \mathrm{mmol} / \mathrm{L}$ (a)、5 $\mathrm{mmol} / \mathrm{L}(\mathrm{b}) 、 45 \mathrm{mmol} / \mathrm{L}(\mathrm{c})$ 氨基甲酸甲酯后, 电沉积 $3 \mathrm{~min}$ 后获得 $\mathrm{Al}$ 沉积层 SEM 图

Figure 3 SEM images of the $\mathrm{Al}$ deposits from $[\mathrm{Emim}] \mathrm{Cl} / \mathrm{AlCl}_{3}$ (33.3/66.7 mol\%) containing (a) no additives, (b) $5 \mathrm{mmol} / \mathrm{L}$ and (c) 45 $\mathrm{mmol} / \mathrm{L}$ methyl carbamate at $1.4 \mathrm{~A} / \mathrm{dm}^{2}, t=3 \mathrm{~min}$

为了研究氨基甲酸甲酯对 $\mathrm{Al}$ 沉积层晶相结构和晶 面趋向的影响, 对加入不同浓度的氨基甲酸甲酯所得的 $\mathrm{Al}$ 沉积层进行 X-射线衍射分析(XRD). 如图 5 所示, 可 以看出, 从纯[ $\mathrm{Emim}] \mathrm{Cl} / \mathrm{AlCl}_{3}(33.3 / 66.7 \mathrm{~mol} \%$ )中得到的 $\mathrm{Al}$ 沉积层呈现出 $\mathrm{Al}(220)$ 晶面择优取向, 但其他晶面峰 强度也相对较高, 所以晶粒形貌呈多面体结构. 加入添 加剂后, $\mathrm{Al}(220)$ 晶面峰强度会随着浓度的提高而降低, 但对 $\mathrm{Al}(311)$ 晶面强度影响较小. 加入 $5 \mathrm{mmol} / \mathrm{L}$ 氨基甲 酸甲酯后, 晶面择优取向为 $\mathrm{Al}(111)$ 和 $\mathrm{Al}(200)$ 同时生长. 提高添加剂浓度, $\mathrm{Al}(111)$ 晶面强度减弱, $\mathrm{Al}(200)$ 晶面强 度增强, 呈现单一晶面择优取向, 所以可以得到平滑致 密的 $\mathrm{Al}$ 沉积层, 这与 SEM 获得的结果是一致的.
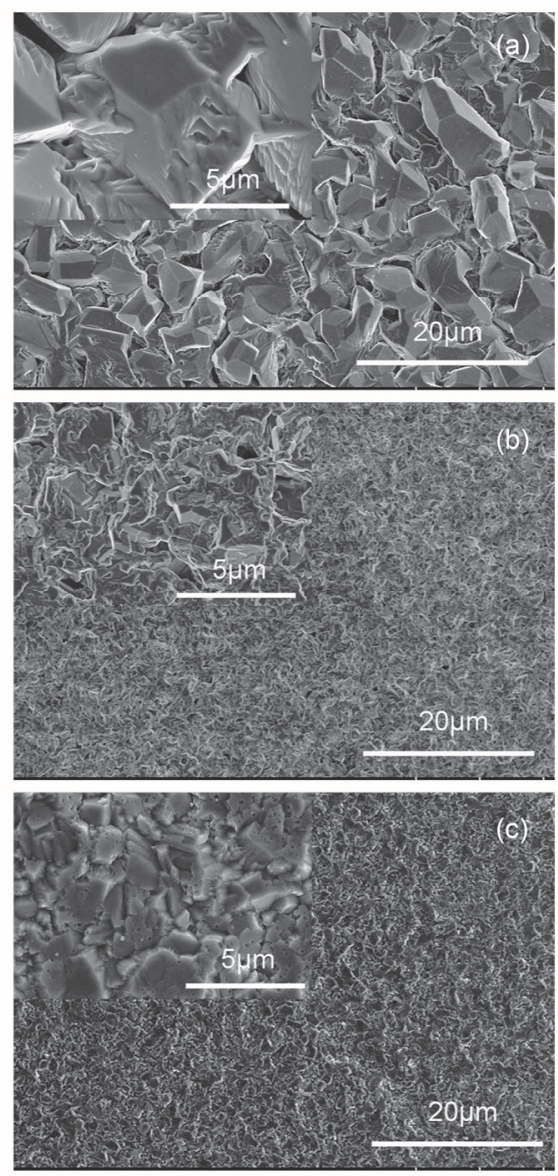

图 4 在 $0.7 \mathrm{~A} / \mathrm{dm}^{2}$ 的电流密度下, 加入不同浓度 $0 \mathrm{mmol} / \mathrm{L} 、 5 \mathrm{mmol} / \mathrm{L}$ 、 $45 \mathrm{mmol} / \mathrm{L}$ 氨基甲酸甲酯后, 电沉积 $8000 \mathrm{~s}$ 后获得 $\mathrm{Al}$ 沉积层 SEM 图 Figure 4 SEM images of the $\mathrm{Al}$ deposits from [Emim]Cl/ $\mathrm{AlCl}_{3}$ (33.3/66.7 mol\%) containing (a) no additives, (b) $5 \mathrm{mmol} / \mathrm{L}$ methyl carbamate and (c) $45 \mathrm{mmol} / \mathrm{L}$ methyl carbamate at $0.7 \mathrm{~A} / \mathrm{dm}^{2}, t=8000 \mathrm{~s}$

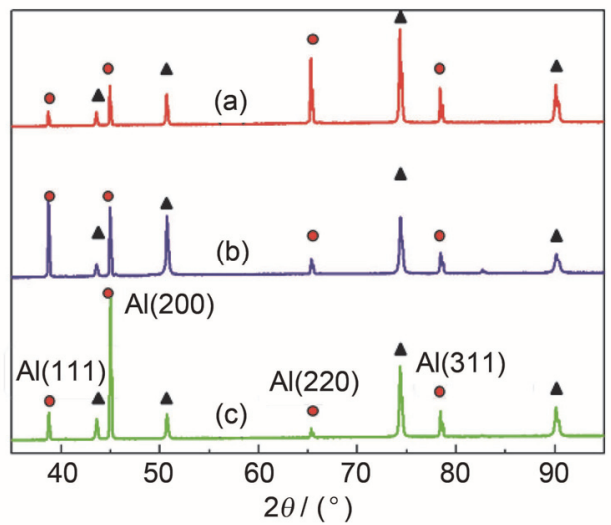

图 5 加入不用浓度氨基甲酸甲酯所得 $\mathrm{Al}$ 沉积层的 XRD 图. (a) 0 $\mathrm{mmol} / \mathrm{L}$, (b) $5 \mathrm{mmol} / \mathrm{L}$, (c) $45 \mathrm{mmol} / \mathrm{L}, \boldsymbol{\Delta}$ 为 $\mathrm{Cu}$ 基底的衍射峰

Figure $5 \mathrm{XRD}$ images of the $\mathrm{Al}$ deposits from $[\mathrm{Emim}] \mathrm{Cl} / \mathrm{AlCl}_{3}$ (33.3/66.7 mol\%) containing (a) $0 \mathrm{mmol} / \mathrm{L}$, (b) $5 \mathrm{mmol} / \mathrm{L}$ methyl carbamate and (c) $45 \mathrm{mmol} / \mathrm{L}$ methyl carbamate at $0.7 \mathrm{~A} / \mathrm{dm}^{2}, t=8000 \mathrm{~s}$

\section{3 氨基甲酸甲酯对电解液中活性铝离子的影响}

$[\mathrm{Emim}] \mathrm{Cl} / \mathrm{AlCl}_{3}(33.3 / 66.7 \mathrm{~mol} \%)$ 离子液体中主要存 在 $\mathrm{Al}_{2} \mathrm{Cl}_{7}^{-}$络合离子, 还有少量的 $\mathrm{AlCl}_{4}^{-}$络合离子, 图 6 
是以二氯甲烷为溶剂, 溶液浓度为 $0.01 \mathrm{~mol} / \mathrm{L}$ 的紫外可 见吸收光谱, $\mathrm{a}$ 曲线是电沉积 $24 \mathrm{~h}$ 后无添加剂离子液体 谱图, b 曲线为加入添加剂电沉积 $24 \mathrm{~h}$ 后离子液体谱图. 从图 6 中可以明显的看出: 两条谱线中均有 $232 \mathrm{~nm}$ 处和 $306 \mathrm{~nm}$ 处存在吸收峰. $232 \mathrm{~nm}$ 处吸收峰, 由咪唑阳离子 环产生. 结合晶体场理论, $306 \mathrm{~nm}$ 处的吸收峰是由络合 离子产生 ${ }^{[26]}$. 由此可以推断氨基甲酸甲酯作为添加剂 时, 在电解液体系中没有形成新的金属络合离子, 不影 响电解液中活性铝离子结构. a 谱线在 $290 \mathrm{~nm}$ 处峰形有 波动, 但 $\mathrm{b}$ 谱线没有类似现象, 表明氨基甲酸甲酯添加 剂的加入对离子液体的咪唑环阳离子具有较好的稳定 作用.

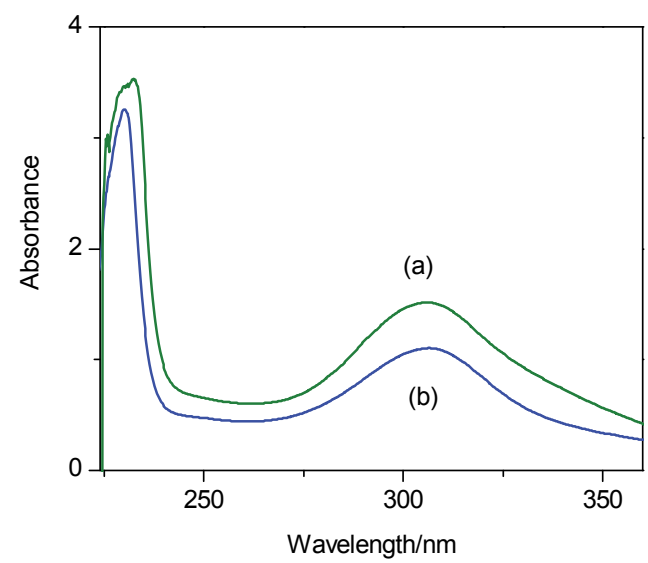

图 6 加入氨基甲酸甲酯与纯 $[\mathrm{Emim}] \mathrm{Cl} / \mathrm{AlCl}_{3}(33.3 / 66.7 \mathrm{~mol} \%$ )紫外可 见吸收图谱

Figure $6 \mathrm{UV}$-Vis spectra of the $\mathrm{Al}$ deposits from $[\mathrm{Emim}] \mathrm{Cl} / \mathrm{AlCl}_{3}$ (33.3/66.7 mol\%) containing (a) $0 \mathrm{mmol} / \mathrm{L}$ and (b) methyl carbamate

核磁测试前, 要在手套箱的惰性气氛下将电解质封 装在核磁管中, 然后进行测试. 溶剂为 D-二氯甲烷, 溶 质氨基甲酸甲酯与 $[\mathrm{Emim}] \mathrm{Cl} / \mathrm{AlCl}_{3}$ 物质的量比为 $1: 1$, 使添加剂与电解质充分结合. 由图 7 可以看出, 氨基甲 酸甲酯加入前后, 体系的 ${ }^{27} \mathrm{Al}$ 核磁谱图中 $\mathrm{Al}$ 络合金属 离子的化学位移没有明显变化. ${ }^{27} \mathrm{Al}$ 核磁谱图与紫外测 试结果一致, 且更加准确地证明了氨基甲酸甲酯的加入 对电解质中的 $\mathrm{Al}(\mathrm{III})$ 离子的络合形态没有影响. 因此, 氨基甲酸甲酯添加剂对电沉积铝层的整平和光滑作用 主要是其在电极表面具有合适的吸附和脱附速率引起 的 ${ }^{[28]}$.

铝在阴极的电沉积一般包括铝的三维成核和生长, 在许多文献中已建立了许多模型来描述电沉积过程. 其 中, 引用较多的是晶核半球式扩散生长模型. 该模型有 两个极限状态, 分别是瞬时成核和连续成核. 这两个过 程可以通过下式来进行描述.

$$
\begin{aligned}
& \left(i / i_{\mathrm{m}}\right)^{2}=1.9542\left(t / t_{\mathrm{m}}\right)^{-1}\left\{1-\exp \left[-1.2564\left(t / t_{\mathrm{m}}\right)\right]\right\}^{2} \\
& \left(i / i_{\mathrm{m}}\right)^{2}=1.2254\left(t / t_{\mathrm{m}}\right)^{-1}\left\{1-\exp \left[-2.3367\left(t / t_{\mathrm{m}}\right)^{2}\right]\right\}^{2}
\end{aligned}
$$

为了研究氨基甲酸甲酯在 $[\mathrm{Emim}] \mathrm{Cl} / \mathrm{AlCl}_{3}$ $(33.3 / 66.7 \mathrm{~mol} \%)$ 离子液体中的电沉积铝过程, 把测得
的电流一时间暂态曲线进行处理，并与上述两个经验模 型进行对比分析 ${ }^{[29,30]}$. 列出 $\left(i / i_{\mathrm{m}}\right)^{2}$ 对 $\left(t^{\prime} / t_{\mathrm{m}}^{\prime}\right)$ 作图的结果和 瞬时成核、连续成核的经验曲线, 如图 8 所示可以看出, 当氨基甲酸甲酯作为添加剂时，铝在阴极的电沉积仍然 符合典型的三维瞬时成核过程，并受物质扩散的影响.

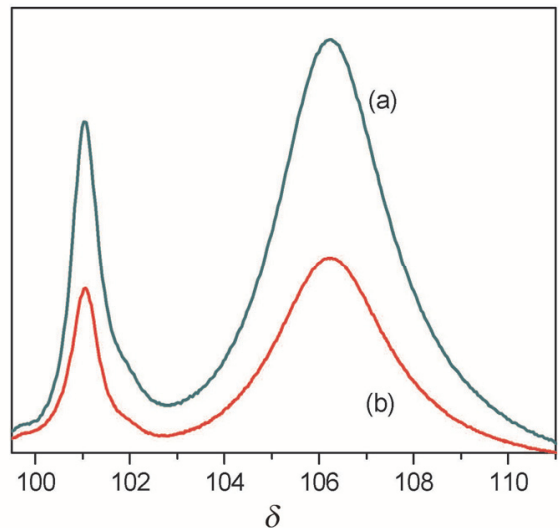

图 7 加入氨基甲酸甲酯前(a)和(b), $[\mathrm{Emim}] \mathrm{Cl} / \mathrm{AlCl}_{3}(33.3 / 66.7 \mathrm{~mol} \%)$ 的 ${ }^{27} \mathrm{Al}$ 核磁谱

Figure $7{ }^{27} \mathrm{Al} \mathrm{NMR}$ spectra of the $\mathrm{Al}$ deposits from [Emim] $\mathrm{Cl} / \mathrm{AlCl}_{3}$ (33.3/66.7 mol\%) containing (a) $0 \mathrm{mmol} / \mathrm{L}$ and (b) methyl carbamate

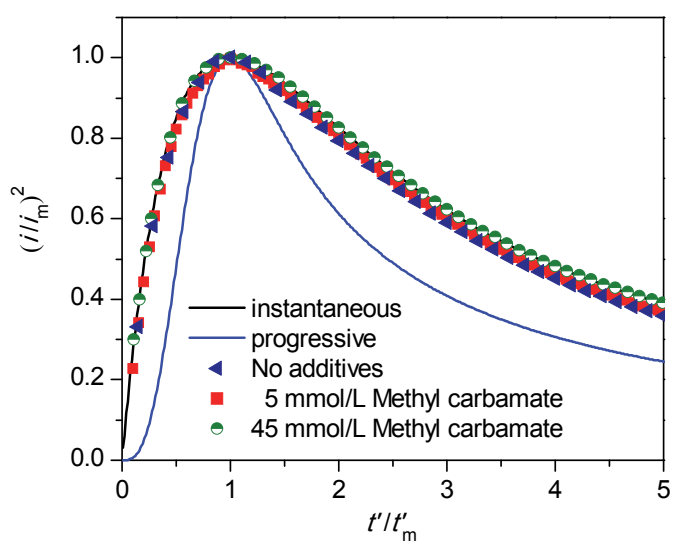

图 8 无因次添加剂电流-时间暂态数据及瞬时成核和连续成核理论 曲线

Figure 8 Non-dimensional plots of $\left(i / i_{\mathrm{m}}\right)^{2}$ vs. $t^{\prime} / t_{\mathrm{m}}^{\prime}$

丙酮和乙酰胺添加剂会参与电沉积反应，同时这种 反应是不可逆的，因此丙酮和乙酰胺不适合作为离子液 体添加剂. 在氨基甲酸甲酯中, 氨基与羰基形成共轭结 构, 形成一个较强的吸电子基团, $\mathrm{C}-\mathrm{O}$ 单键也是一个吸 电子基团. 因此羰基的 $\mathrm{C}$ 原子作为中心原子，电子云因 其他官能团的影响受到迁移, 中心原子 $\mathrm{C}$ 显较强的正电 性. 而乙酸和乙酸乙酯的吸电子能力较弱, 吸附在阴极 表面的能力较弱. 但氨基甲酸甲酯有较好的吸附性，易 于吸附在阴极表面, 抑制 $\mathrm{Al}$ 的电沉积反应, 增加电化学 极化, 提高沉积过电位.

\section{3 结论}

(1) 添加剂的引入, 使得电沉积铝的过电位增加, 
沉积电位升高, 沉积晶粒变小.

（2）氨基甲酸甲酯是一种性能优异的 $\mathrm{Al}$ 沉积整平 添加剂, 随着添加剂浓度的提高即可明显改善阴极 $\mathrm{Al}$ 沉积层的光亮度, 沉积层的颗粒尺寸逐渐减小, 得到非 常细致均匀光滑且镜面光亮的 $\mathrm{Al}$ 沉积层, 呈现较强的 $\mathrm{Al}(200)$ 晶面取向.

(3) 通过循环伏安测试并结合紫外可见吸收光谱、 ${ }^{27} \mathrm{Al}$ 核磁分析, 发现氨基甲酸甲酯作为添加剂在电极表 面具有良好的吸附能力, 在没有形成新的金属络合离子 的条件下，一方面可以对离子液体阳离子产生一定的保 护作用, 另一方面可以对 Al 的电沉积过程产生抑制作 用，从而使沉积层晶粒细化且均匀致密.

（4）氨基甲酸甲酯在电极表面良好的吸附作用主要 取决于含羰基类添加剂的中心 $\mathrm{C}$ 原子上所连的 2 个取代 基的性质, 这对离子液体电沉积体系添加剂的选择具有 重要的指导意义.

\section{4 实验部分}

\section{1 实验材料}

1-乙基-3-甲基咪唑氯盐([Emim]Cl)购于林州科能材 料科技有限公司, 纯度 $>99.7 \%$; 无水三氯化铝购于国 药集团化学试剂有限公司(上海), 纯度 $99 \%$. [Emim] Cl 在 $353 \mathrm{~K}$ 利用减压蒸馏装置(自制)干燥 $24 \mathrm{~h}$, 干燥后水 分含量低于 $15 \mathrm{mg} / \mathrm{L}$, 并置于浓度为 $99.9 \%$ 的 $\mathrm{Ar}$ 气手套 箱中保存. 将 [Emim] Cl 与 $\mathrm{AlCl}_{3}$ 以物质的量比 $1: 2$ 的比 例在手套相中缓慢混合, 得到无色透明溶液. 以高纯 $\mathrm{Al}$ 板 $(99.99 \%)$ 作为阴、阳电极, 在温度 $323 \mathrm{~K}$, 电压 $1.08 \mathrm{~V}$ 直流电压下进行预电解 $48 \mathrm{~h}$. 添加剂: 丙酮、乙酰胺、 乙酸、乙酸甲酯均购于国药集团化学试剂有限公司(上 海), AR. 氨基甲酸甲酯购于梯希爱(上海)化成工业发展 有限公司，纯度 $98 \%$.

\section{2 实验方法}

循环伏安曲线利用 CHI660E 电化学工作站(上海辰 华仪器有限公司) 获得. 玻碳(直径 $2 \mathrm{~mm}$ ) 电极为工作电 极, 纯度为 $99.99 \%$ 的 $\mathrm{Al}$ 片 $(5 \mathrm{~mm} \times 5 \mathrm{~mm} \times 0.5 \mathrm{~mm})$ 为对 电极, $\mathrm{Al}$ 丝(纯度 $99.99 \%$ )为参比电极. 利用恒电流方法 在 $[\mathrm{Emim}] \mathrm{Cl} / \mathrm{AlCl}_{3}(33.3 / 66.7 \mathrm{~mol} \%)$ 中获得铝沉积层, 阳 极为铝板 $(13 \mathrm{~mm} \times 33 \mathrm{~mm} \times 1 \mathrm{~m}, 99.99 \%)$, 阴极为铜片 $(10 \mathrm{~mm} \times 30 \mathrm{~mm} \times 0.3 \mathrm{~m}, 99 \%), \mathrm{Al}$ 丝为参比电极. 实验 结束后用体积比 $1: 1$ 的乙醇和丙酮的混合液清洗样片, 再用蒸馏水清洗干净, 氮气吹扫干燥.

\section{3 分析方法}

采用紫外可见分光光度计(Labtech UV2000, 美国) 分析电沉积实验后电解液的组成变化. 采用核磁共振波
谱仪(Bruker AV-500 MHz，瑞士)以氝代二氯甲烷为溶剂 进行 ${ }^{27} \mathrm{Al}$ 谱图测试, 分析加入添加剂后电解液组成和键 合变化规律. 采用冷场发射扫描电子显微镜与能谱分析 仪(SEM, 日立 SU8020, 日本)对铝沉积层形貌进行观 察. 采用 X 射线衍射仪(XRD, Smartlab, Rigaku Ltd. 日 本)对铝沉积层进行物相分析.

\section{References}

[1] Chang, J. K.; Chen, S. Y.; Tsai, W. T.; Deng, M. J.; Sun, I. W. Electrochem. Commun. 2007, 9(7), 1602.

[2] Endo, A.; Miyake, M.; Hirato, T. Electrochim. Acta 2014, 137, 470.

[3] NuLi, Y. N.; Du, G. D.; Feng, Z. Z.; Shen, J.-N.; Yang, J. Acta Chim. Sinica 2008, 66, 175. (努丽燕娜, 杜国栋, 冯真真, 沈佳妮, 杨军, 化学学报, 2008, 66, 175.)

[4] NuLi, Y. N.; Yang, J.; Gao, P. F.; Li, Y.; Wang, J. L. Acta Chim. Sinica 2010, 68, 948. (努丽燕娜, 杨军, 高鹏飞, 李云, 王久林, 化学学报, 2010, 68,948 .)

[5] Hurley, F. H.; Wier, T. P. J. Electrochem. Soc. 1951, 85(5), 207.

[6] Liao, Q.; Pitner, W. R.; Stewart, G.; Hussey, C. L.; Stafford, G. R. J. Electrochem. Soc. 1997, 144(3), 936.

[7] Lee, J. J.; Bae, I. T.; Scherson, D. A.; Miller, B.; Wheeler, K. A. J. Electrochem. Soc. 2000, 147(2), 562.

[8] Liu, Q. X.; El. Abedin, S. Z.; Endres, F. Surf. Coat. Technol. 2006, $201,1352$.

[9] Tang, J. W.; Azumi, K. Electrochim. Acta 2011, 56, 1130.

[10] Zhao, Y. G.; VanderNoot, T. J. Electrochim. Acta 1997, 42(11), 1639.

[11] Chang, J. K.; Chen, S. Y.; Tsaia, W. T.; Deng, M. J.; Sun, I. W. J. Electrochem. Soc. 2008, 155(3), C112.

[12] Yue, G. K.; Zhang, S. J.; Zhu, Y. L.; Lu, X. M.; Li, S. C.; Li, Z. X. AIChE J. 2009, 55, 783 .

[13] Jiang, T.; Brym, M. J. C.; Dube, G.; Lasia, A.; Brisard, G. M. Surf. Coat. Technol. 2006, 201, 10.

[14] Su, C. J.; Hsieh, Y. T.; Chen, C. C. Electrochem. Commun. 2013, 34, 170.

[15] El. Abedin, S. Z.; Moustafa, E. M.; Hempelmann, R.; Natter, H.; Endres, F. Electrochem. Commun. 2005, 7, 1111.

[16] El. Abedin, S. Z.; Moustafa, E. M.; Hempelmann, R.; Natter, H.; Endres, F. ChemPhysChem 2006, 7(11), 1535.

[17] Moustafa, E. M.; El. Abedin, S. Z.; Shkurankov, A.; Zschippang, E.; Saad, A. Y.; Bund, A.; Endres, F. J. Phys. Chem. B 2007, 111, 4693.

[18] Atkin, R.; El. Abedin, S. Z.; Hayes, R.; Gasparotto, L. H. S.; Borisenko, N.; Endres, F. J. Phys. Chem. C 2009, 113, 13266.

[19] Giridhar, P.; El. Abedin, S. Z.; Endres, F. Electrochim. Acta 2012, $70,21$.

[20] Zheng, Y.; Dong, K.; Wang, Q.; Zhang, S. J.; Zhang, Q. Q.; Lu, X. M. Sci. China Chem. 2012, 55(8), 1587 .

[21] Zheng, Y.; Zhang, S. J.; Lu, X. M.; Wang, Q.; Zuo, Y.; Liu, L. Chinese J. Chem. Eng. 2012, 20(1), 130.

[22] Abbott, A. P.; Qiu, F.; Abood, H. M. A.; Ali, M. R.; Ryder, K. S. Phys. Chem. Chem. Phys. 2010, 12, 1862.

[23] Liu, L.; Lu, X. M.; Cai, Y. J.; Zheng, Y.; Zhang, S. Z. Aust. J. Chem. 2012, 65(11), 1523 .

[24] Li, B.; Fan, C. H.; Chen, Y.; Lou, J. W.; Yan, L. G. Electrochim. Acta 2011, 56(16), 5478.

[25] Barchi, L.; Bardi, U.; Caporali, S.; Fantini, M.; Scrivani, A. Prog. Org. Coat. 2010, 68, 120

[26] Endres, F.; Bukowski, M.; Hempelmann, R.; Natter, H. Angew. Chem. Int. Ed. 2003, $42,3428$.

[27] Zhang, Q. Q.; Wang, Q.; Zhang, S. Z.; Lu, X. M. J. Solid State Electrochem. 2014, 18(1), 257.

[28] Wang, X. M. Ph.D. Dissertation Shandong University of Technology, Zibo, 2010. (王晓铭, 博士论文, 山东理工大学, 淄博, 2010.)

[29] Zhu, Y. L.; Katayama, Y.; Miura, T. Electrochim. Acta 2012, 85(4), 622.

[30] Zhu, Y. L.; Katayama, Y.; Miura, T. Electrochim. Acta 2010, 55(28), 9019. 